\title{
Platelet Rich Fibrin in the Treatment of Localised Gingival Recession: A Case Report
}

\author{
Dhawal Mody ${ }^{1}$, Vrushali Lathiya ${ }^{2}$ \\ ${ }^{1}$ Associate Professor, ${ }^{2}$ Senior Lecturer, \\ VSPM Dental College \& Research Centre, Department of Periodontology, \\ Digdoh Hills, Hingna Road, Nagpur - 440019 \\ Corresponding Author: Dhawal Mody
}

\begin{abstract}
Root recession presents a matter of aesthetic concern in every dental practice. Also it may illicit sensitivity and is more prone to caries than the covered root surfaces. Platelet- rich fibrin is a second generation platelet concentrate. It is basically autologous leukocyte and platelet-rich fibrin biomaterial. The use of PRF in combination with coronally advanced flap in the treatment of gingival recession has shown promising results and is gaining immense popularity among the dental practitioners. This case reports the use of PRF membrane in root coverage on the labial surface of maxillary canine.
\end{abstract}

Key words: Coronally advanced flap, platelet rich fibrin, gingival recession, growth factors

\section{INTRODUCTION}

Gingival recession (GR) is the exposure of the root surface due to displacement of the gingival margin apical to the cement-enamel junction (CEJ). ${ }^{[1,2]}$ The etiologic factors associated with the gingival recession include mechanical trauma, improper tooth brushing, orthodontic appliances, periodontal diseases, occlusal trauma, tooth malpositioning, orthodontic tooth movement, etc. ${ }^{[3]}$ Amongst the various root coverage procedure that have been developed to treat gingival recessions, Coronally Advanced Flap (CAF) with connective tissue graft (CTG) is usually considered as a gold standard and is the most predictable surgical procedure for complete coverage in Millers Class I \& Class II gingival recession. ${ }^{[4]}$

Obtaining an optimal root coverage and acceptable esthetics is the primary aim of mucogingival surgeries. But when selecting the surgical technique care should also be taken regarding patient comfort. Several regenerative materials are also being used in combination with CAF. One such regenerative material is autologous platelet concentrates. ${ }^{[5]}$ Platelet- rich fibrin (PRF) is a second-generation platelet concentrate and it was first described by Choukroun et al. [6] It is the latest development amongst all the platelet derived products and techniques. Plateletrich plasma (PRP) was the first generation scaffold derived from blood samples of humans but the clinical applications of PRP were limited due to addition of anticoagulants and bovine serum and double centrifugation. $^{[7]}$ On the contrary no anticoagulants, thrombin or calcium chloride are required in the preparation of PRF. PRF is rich in fibronectin and releases various growth factors that promote regeneration. It has a great potential for hard tissue and soft tissue regeneration. Therefore taking into consideration, the numerous advantages of platelet rich fibrin, this case reports the use of PRF membrane in combination with CAF in the treatment of localised gingival recession. 


\section{CLINICAL PRESENTATION}

A 23 year old female reported to the Dept. of Periodontics, of our institute with a chief complaint of poor esthetics in the upper left anterior region since six months. On clinical examination of the patient, a Class I Miller's gingival recession was found in association with 23 . The UNC -15 probe revealed $6 \mathrm{~mm}$ of clinical attachment loss (CAL). The amount of gingival recession (GR) measured as the distance between the cementoenamel junction (CEJ) and free gingival margin was $4 \mathrm{~mm}$ and the Probing pocket depth (PPD) measured as the distance between the gingival margin to the base of the pocket was $2 \mathrm{~mm}$ (Fig. 1a).
There was an adequate width of attached gingival and slight bleeding on probing. After a thorough clinical examination it was decided to treat the gingival recession by employing a coronally advanced flap in combination with PRF. The surgical procedure was explained and the informed consent was obtained from the patient. Scaling and Root Planing of the entire dentition was done and oral hygiene instructions were given. Probing pocket depth (PPD), gingival recession and width of attached gingiva were recorded before and after the mucogingival surgical procedure.

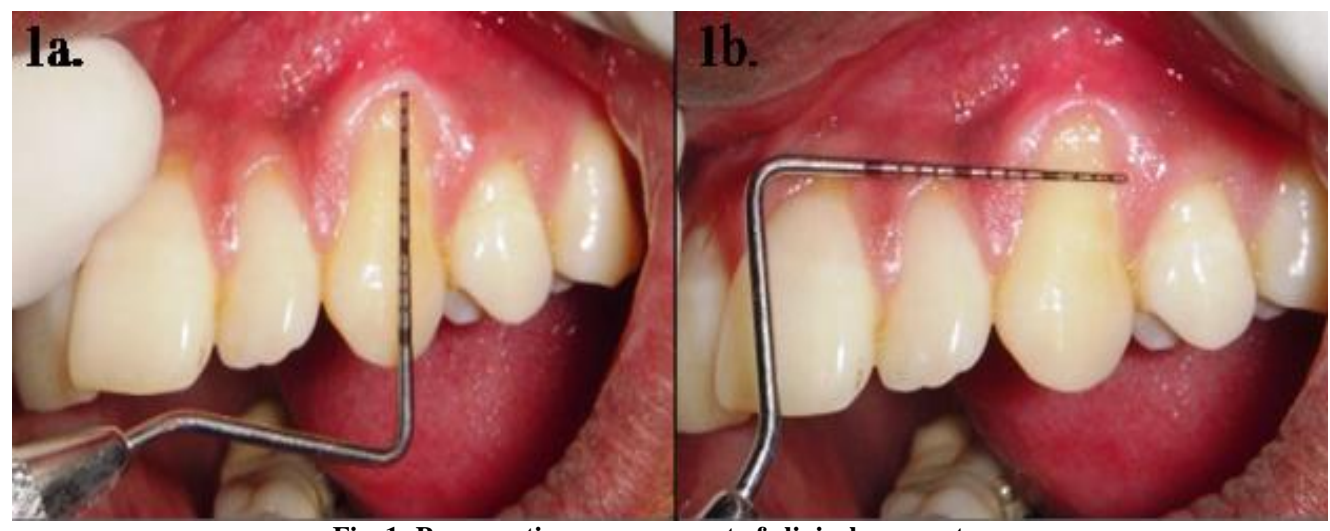

Fig. 1: Preoperative measurement of clinical parameters.

1a) Vertical measurement of Gingival recession (GR) defect in $\mathrm{mm}$

1b) Horizontal measurement of Probing Pocket depth (PPD) in mm

\section{Treatment Strategy}

\section{Preparation of Platelet Concentrate}

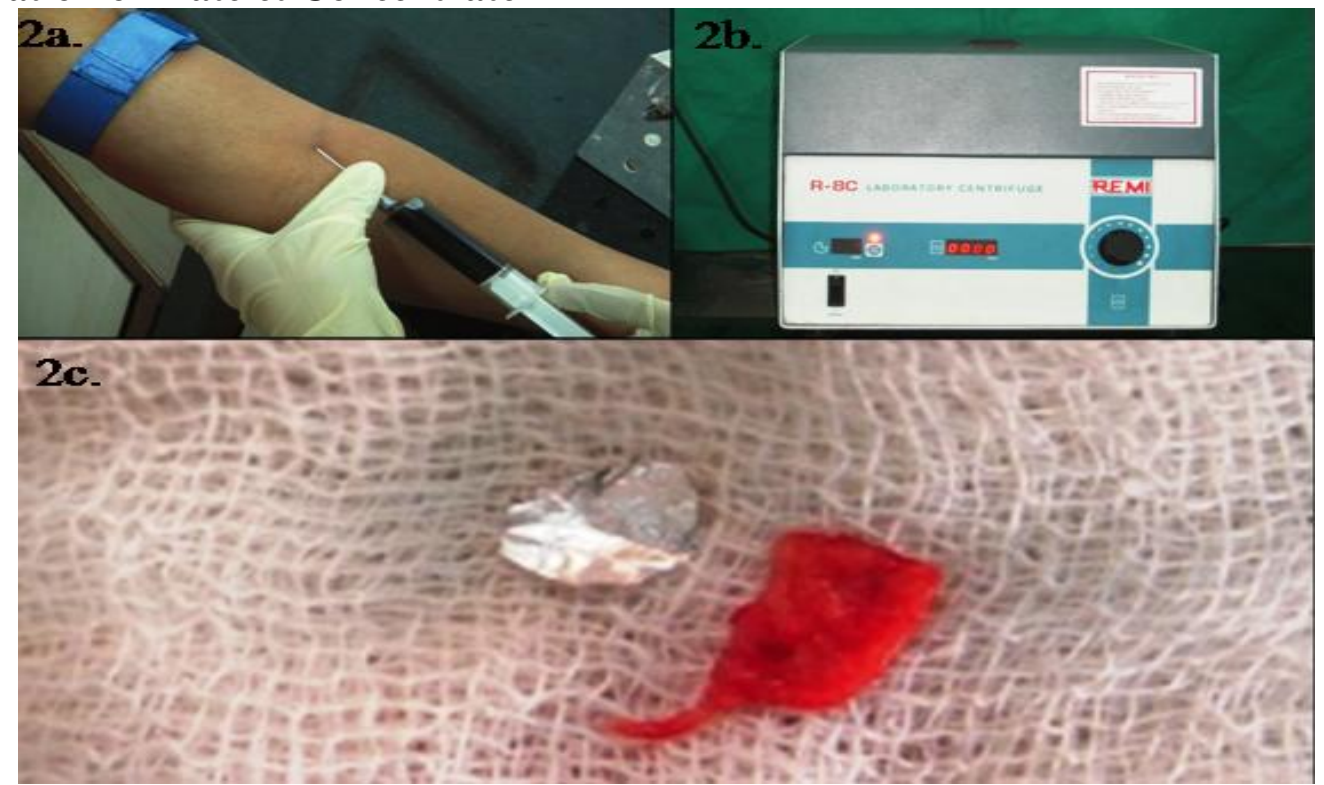

Fig. 2: Preparation of Platelet Concentrate.

2a) Drawing of blood from the antecubital vein.

2b) Centrifugation of the blood contained in the test tubes.

2c) Preparation of PRF membrane 
Platelet rich fibrin was prepared according to Choukroun et al. protocol ${ }^{[8]} 10$ $\mathrm{ml}$ of blood was drawn from each patient immediately prior to the surgical procedure by venipuncture of the antecubital vein and collected in a sterile glass test tube without any anticoagulant (Fig.2a) Because of the absence of an anticoagulant, the blood was quickly collected, and the tubes were immediately centrifuged for 12 minutes at 2,700 rpm (Fig. 2b). The topmost layer consisted of acellular platelet-poor plasma (PPP), a PRF clot in the middle, red blood cells (RBCs) were formed at the base. PRF clot was procured in the form of a membrane by compressing out the fluids from the PRF clot and placed in a sterile petri dish. ${ }^{[9]}$ (Fig.2c)

\section{Preparation of the Recipient Site}

Surgical field was properly isolated; the operative sites were anaesthetized using $2 \%$ per cent xylocaine hydrochloride with adrenaline (1:200000). The surgical procedure was done as described by Allen and Miller in 1989. [10] The coronally repositioned flap procedure was performed. On the buccal aspect of the maxillary canine, intrasulcular incision was given followed by horizontal incisions perpendicular to the interdental papillae which is adjacent to the affected site (Fig. 3a). A moderate to deep vestibule is essential as it allows coronal displacement of flap without tension. A full-thickness flap was reflected till the mucogingival junction and was further released by sharp dissection. (Fig. 3b) The mesiodistal length of the incision was extended to the nearest distal line angle of the most mesial and distal teeth involved. Releasing incisions across the MGJ were avoided during the initial phase of the surgical procedure to reduce postoperative swelling and pain. At the same time, the papilla over which the CAF was to be placed was de-epithelised to create a connective tissue bed. The PRF membrane was then placed over the denuded root surface at the recipient site and then stabilized with the help of (5-0) bio-absorbable sling suture (Ethicon, St.Stevens-Woluwe, Belgium). The flap was then coronally advanced to completely cover the membrane and sutured over the graft with sling sutures (Mersilk 4-0) (Fig. $3 c)$. Vertical incisions were also closed with 4-0 silk sutures. Finally, it was covered with periodontal dressing and mild pressure was applied for $5 \mathrm{~min}$ to minimize dead space.

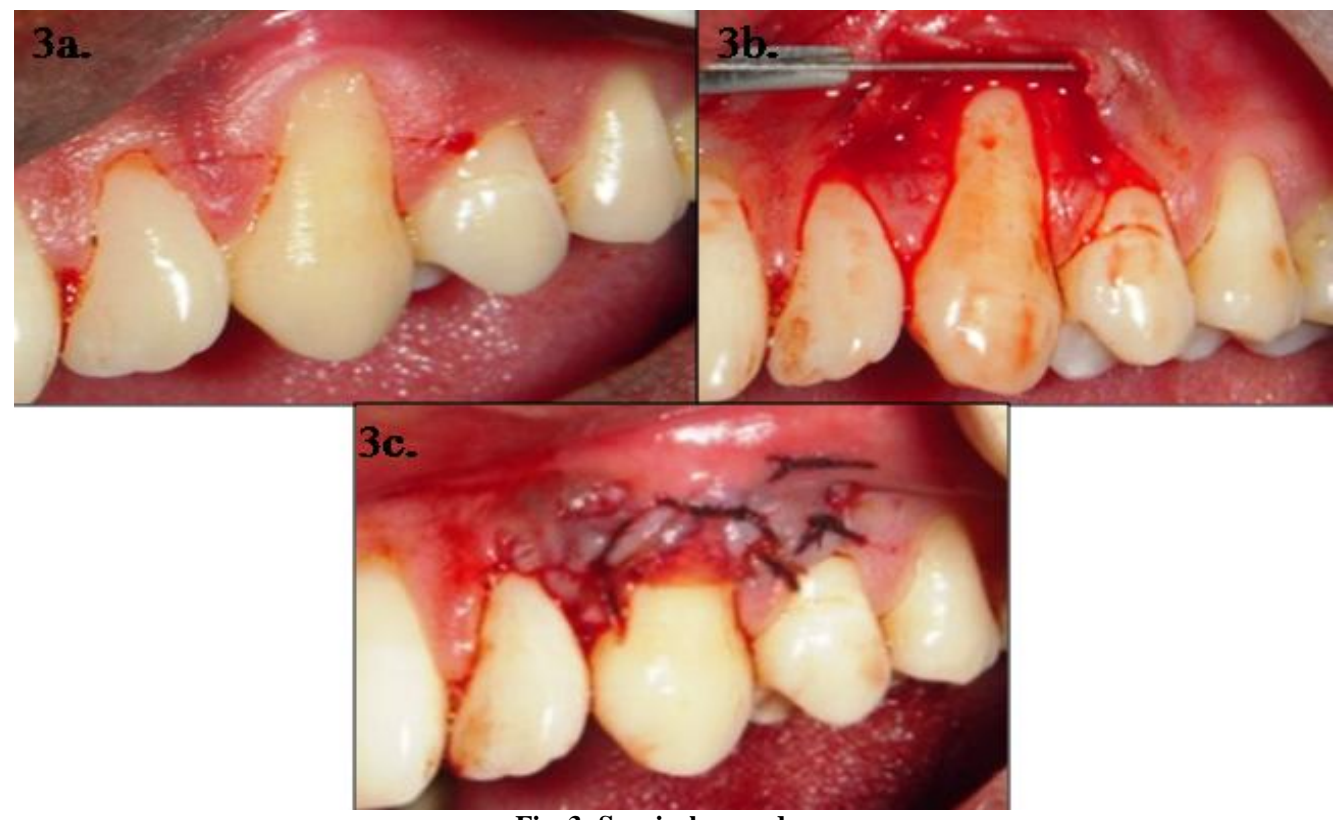

Fig. 3: Surgical procedure.

3a) Horizontal and sulcular incision placement on the buccal aspect of 23

3b) Flap reflection beyond the mucogingival junction 3c) Placement of sutures 


\section{Post-Operative care}

The patient was advised to use $0.2 \%$ chlorhexidine digluconate mouthrinse, twice daily for two weeks. Systemic analgesics were prescribed and advised to follow routine post-operative periodontal instructions. The dressings and sutures were removed 10 days after the surgery. There was almost $100 \%$ root coverage at the time of suture removal. (Fig. 4a) The patient was revaluated after 6 months. The results persisted even at the end of 6 months and there was a significant increase in the width of attached gingiva. (Fig. 4b)

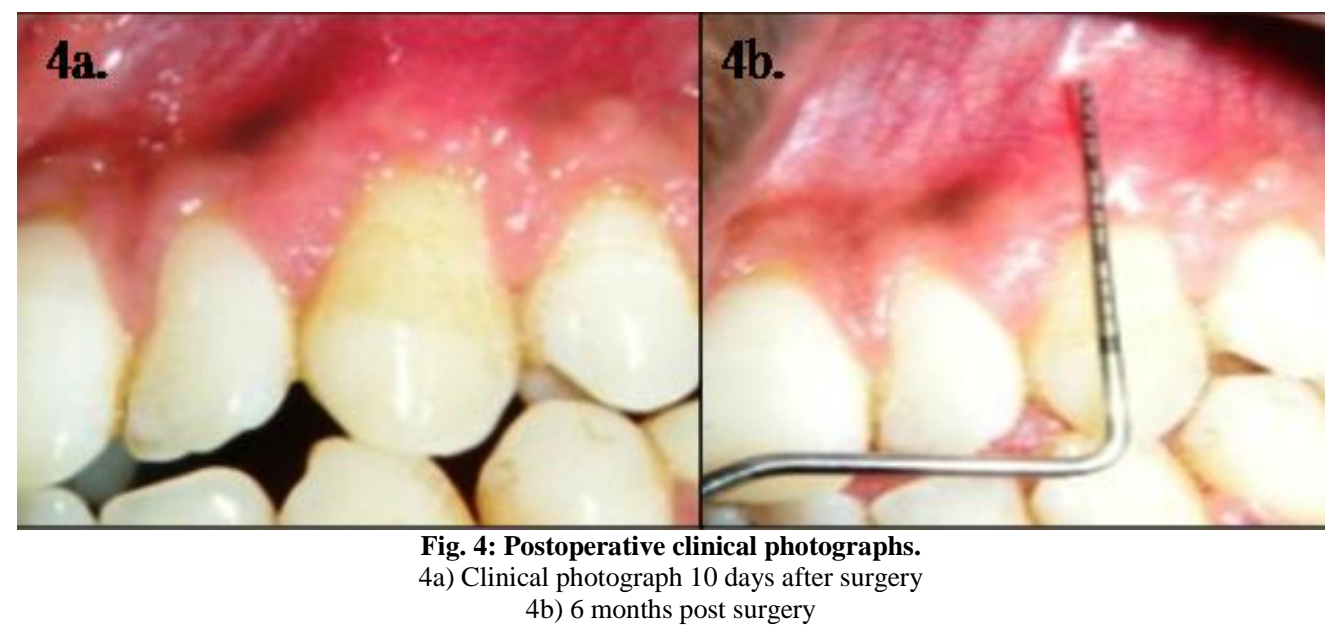

\section{DISCUSSION}

An increasing awareness regarding aesthetics in the general population has substantially risen the demand for periodontal plastic and esthetic procedures. The selection of an ideal site is an extremely crucial factor when it comes to determining the prognosis of root coverage procedures. Millers Class I and Class II gingival recession are considered as ideal candidates for root coverage wherein one can expect almost $100 \%$ of recession coverage. In the present case report we treated a Class I gingival recession present on the buccal aspect of 23 with PRF in combination with CAF. The need for donor site procurement of connective tissue and unnecessary trauma to the patient was avoided in our case report by using PRF membrane. Choukroun's PRF has proven to be a method that comes close to the ideal concept of guided ("smart") tissue engineering over the last few years. As previously shown, this form of PRF contains various growth factors and can contribute to tissue regeneration in terms of osteoblast, prekeratinocyte, and gingival fibroblast differentiation and promoting neoangeogenesis. ${ }^{[11]}$ Platelet alpha-granules are a reservoir of many growth factors that are known to play a crucial role in hard and soft tissue repair and regeneration mechanisms. ${ }^{[12,13]}$ These include plateletderived growth factors (PDGFs), transforming growth factor-beta (TGF-beta), vascular endothelial growth factor (VEGF), epidermal growth factor (EGF), and insulinlike growth factor-1 (IGF-1). These growth factors enhance the chemotatic and mitotic activity and stimulate the migration and proliferation of connective tissue.

The above case report showed an increase in the width of attached gingiva. These findings are in accordance with the study done by Kuka S. et al. who evaluated coronally advanced flap with or without platelet-rich fibrin for the treatment of multiple gingival recessions and concluded that the use of PRF membranes increased the width of attached gingiva significantly. ${ }^{[14]}$

In yet another study, Del Corso $M$ et al. found significant improvement during the early periodontal healing phases with a thick and stable, final remodelled gingiva. ${ }^{[15]}$ Aroca et al., reported that addition of a PRF membrane positioned 
under the Modified Coronally Advanced Flap provided excellent results when used to treat gingival recession with an additional gain in the gingival at end of 6 months post treatment as compared to conventional therapy. ${ }^{[16]}$ Recently a concept of advanced Platelet-Rich Fibrin has been studied. It is relatively a New Concept for Cell-Based Tissue Engineering by Means of Inflammatory Cells. [7] Also a novel compression device was developed for preparation of PRF membrane. This device minimizes the damage of platelets contained in the PRF membranes and also minimizes the lost or degradation of growth factors in the PRF membranes. ${ }^{[17]}$

This case report shows that the use of platelet rich fibrin in combination with coronally advanced flap enhances the clinical outcome of root coverage. A histologic evaluation is however necessary to study the nature of healing and this could be considered as a limitation of the present case report. Further studies should be conducted directed towards analyzing the type of healing after the root coverage procedures and a long term follow up is essential to substantiate the results.

\section{CONCLUSION}

Platelet Rich Fibrin in the treatment of gingival recession provides a promising approach in the treatment of Millers Class I and Class II gingival recession and can be used as an alternative to Sub Epithelial Connective tissue graft. The advantages of periodontal regeneration and a faster healing have made the use of PRF extremely popular among the dental practitioners. The exact mechanism by which PRF improves the clinical outcome is not very clear but it has a vast scope in the field of periodontal regeneration.

Prior Publication: None

Acknowledgement: None

Conflict of Interest: None

\section{Source of Funding: None}

\section{REFERENCES}

1. Camargo PM, Melnick PR, Kenney EB. The use of free gingival grafts for aesthetic purposes. Periodontol 2000 2001; 27:72-96.

2. Wennström JL, Zucchelli G. Increased gingival dimensions. A significant factor for successful outcome of root coverage procedures? A 2-year prospective clinical study. J Clin Periodontol 1996; 23(8): 7707.

3. Pai JBS., et al. "Modified semilunar coronally advanced flap: A case series". Journal of Indian Society of Periodontology 17.1 (2013): 124-127.

4. Hofmänner $\mathrm{P}$, Alessandri R, Laugisch $\mathrm{O}$, Aroca S, Salvi GE, Stavropoulos A, Sculean A. Predictability of surgical techniques used for coverage of multiple adjacent gingival recessions--A systematic review. Quintessence Int. 2012; 43(7):545-54.

5. Thamaraiselvan M., et al. "Comparative clinical evaluation of coronally advanced flap with or without platelet rich fibrin membrane in the treatment of isolated gingival recession". Journal of Indian Society of Periodontology 19.1 (2015): 6671.

6. David M. Dohan, Joseph Choukroun, Antoine Diss, Steve L. Dohan, Anthony J. J. Dohan, Jaafar Mouhyi, Bruno Gogly. Platelet-rich fibrin (PRF): A secondgeneration platelet concentrate. Part I: Technological concepts and evolution. Oral Surg Oral Med Oral Pathol Oral Radiol Endod 2006; 101:E37-44.

7. Shahram Ghanaati, Patrick Booms, Anna Orlowska, Alica Kubesch, Jonas Lorenz, Jim Rutkowski, Constantin Landes, Robert Sader, CJ Kirkpatrick, Joseph Choukroun, Advanced Platelet-Rich Fibrin: A New Concept for Cell-Based Tissue Engineering by Means of Inflammatory Cells, Journal of Oral Implantology. 2014; 40 (6):679-689.

8. Choukroun J., et al. "An opportunity in perio-implantology: The PRF". Implantodontie 42 (2001): 55-62.

9. Griffin TJ, Cheung WS. Treatment of gingival recession with a platelet concentrate graft: A report of two cases. Int J Periodontics Restorative Dent. 2004; 24:589-95. 
10. Allen EP, Miller PD Jr. Coronal positioning of existing gingiva: Short term results in the treatment of shallow marginal tissue recession. J Periodontol 1989; 60:316-319.

11. Dohan Ehrenfest DM, Diss A, Odin G, Doglioli P, Hippolyte M, Charrier J. In vitro effects of Choukroun's PRF (platelet-rich fibrin) on human gingival fibroblasts, dermal prekeratinocytes, preadipocytes, and maxillofacial osteoblasts in primary cultures. Oral Surg Oral Med Oral Pathol Oral Radiol Endod 2009; 108:341-352.

12. Anitua E, Sanchez M, Orive G, Andia I. The potential impact of the preparation rich in growth factors (PRGF) in different medical fields. Biomaterials 2007; 28:4551-4560.

13. Marx RE, Carlson ER, Eichstaedt RM, Schimmele SR, Strauss JE, Georgeff KR. Platelet-rich plasma: Growth factor enhancement for bone grafts. Oral Surg Oral Med Oral Pathol Oral Radiol Endod 1998; 85:638-646.

14. Kuka S, Ipci SD, Cakar G, Y1lmaz S. Clinical evaluation of coronally advanced flap with or without platelet-rich fibrin for the treatment of multiple gingival recessions. Clin Oral Investig. 2018 Apr; 22 (3):1551-1558.
15. Del Corso M, Sammartino G, Dohan Ehrenfest DM. Clinical evaluation of a modified coronally advanced flap alone or in combination with a platelet-rich fibrin membrane for the treatment of adjacent multiple gingival recessions: A 6-month study. J Periodontol 2009; 80: 1694-1697.

16. Aroca S, Keglevich T, Barbieri B, Gera I, Etienne D. Clinical Evaluation of a Modified Coronally Advanced Flap Alone or in Combination with a Platelet-Rich Fibrin Membrane for the Treatment of Adjacent Multiple Gingival Recessions: A 6-Month Study. J Periodontol 2009; 80:244252.

17. Mito Kobayashi, Tomoyuki Kawase, Makoto Horimizu Kazuhiro Okuda Larry F.Wolff, Hiromasa Yoshie. A proposed protocol for the standardized preparation of PRF membranes for clinical use. Biologicals 2012; 40(5):323-329.

How to cite this article: Mody D, Lathiya V. Platelet rich fibrin in the treatment of localised gingival recession: a case report. International Journal of Research and Review. 2021; 8(5): 24-29. DOI: https://doi.org/10.52403/ijrr. 20210505 